\title{
Smart Privacy Management in Ubiquitous Computing Environments
}

\author{
Christian Bünnig* \\ University of Rostock, Institute of Computer Science \\ Chair for Information and Communication Services \\ christian.buennig@uni-rostock.de
}

\begin{abstract}
Privacy in ubiquitous computing environments is primarily considered as a problem of protecting personal information from unauthorized access and misuse. Additionally it can also be seen as a process of interpersonal communication where not hiding but selective disclosure of personal information is the central issue, i.e. how users can practice privacy intuitively and dynamically in computerized environments similar to the analog world. In this work we discuss the management of private information concerning interpersonal privacy implications in smart environments. Existing work mostly does not match the intuitive and dynamic aspects of privacy in context of interpersonal communication. As an alternative we suggest an ad hoc approach to privacy management which uses learning techniques for an in situ disclosure assistance and present user interaction models for this disclosure assistance.
\end{abstract}

\section{Introduction}

Privacy in ubiquitous computing environments is still an open research issue. In most cases privacy is considered as a problem of protecting personal information to make it only visible to certain entities or from being misused by malicious entities. While this is an important aspect of privacy on the data level it neglects that privacy is much more than hiding personal information. Often people want to show certain information to other entities, e.g. to utilize personalized services, to receive information from other persons in return or to represent oneself to the public in a specific manner. Especially this self-representation in a social context is a dynamic process in which humans intuitively decide which personal face to show depending on the current situation [10. The crucial issue is how ubiquitous computing environments with its potentially vast amount of communicated personal information influences intuitive capabilities to practise privacy. When acting in smart environments the personal information communicated within that environment creates further instances of a user's self-representation which makes privacy management more complex compared to the analog world. Users

\footnotetext{
* Christian Bünnig is funded by the German Research Foundation (DFG), Graduate School 1424 (Multimodal Smart Appliance Ensembles for Mobile Applications MuSAMA).
}

M.J. Smith and G. Salvendy (Eds.): Human Interface, Part II, HCII 2009, LNCS 5618, pp.131-139, 2009. (C) Springer-Verlag Berlin Heidelberg 2009 
need an understanding of the implications of communicating specific personal information. In general there are two types of implications. The first is a technical one, e.g. a possible recording and post processing of the communicated information by the environment's infrastructure. The second relates to interpersonal implications because other persons within the environment directly or indirectly perceive the communicated information. In this work we focus on interpersonal implications and assume a non-malicious infrastructure which handles private data as users expect it. Concerning interpersonal implications, in most cases users intend them as they are the reason for acting within a smart environment, e.g. because they want to collaborate with colleagues or socialize with friends. The point is how users can ensure that actual implications comply with intended ones, or in other words, how users can be enabled to practise interpersonal privacy in smart environments as similar as possible to the way it is done in a non-technical environment.

\section{Example Scenario}

A typical use case for smart environments is the support of collaborative work. Consider the following scenario as an illustrative example. A room is equipped with a collaboration desk - a desk with a touch screen surface used as a shared work space (Fig. 11). Depending on a specific application this desk may display and arrange various information, contributed by the persons working at the desk. A team working on a film project meets at this desk to discuss current results and upcoming tasks in the project. Depending on the

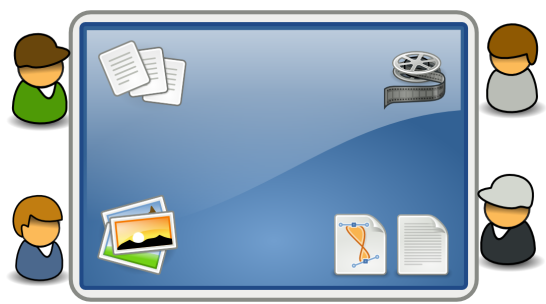

Fig. 1. A collaboration desk as an example service for interpersonal communication in smart environments current state of the project, the persons present at the meeting and each person's task in the project, each team member wants to provide specific information to the meeting by disclosing them to the collaboration desk service. Alice contributes some drafts for a poster, Bob has a story board ready and Clark has some suggestions concerning the crew casting. Dent, the organizer of the team, guides the meeting and controls and arranges the currently displayed information on the desk. Additionally Dent requests to access the calendar of the meeting participants in order to schedule upcoming events. All team members are working as freelancers - they may work at this desk in other projects, in other roles and with other team members too.

Special characteristics of this scenario are that there is no distinct hierarchy among the persons and no fixed relationship between the persons and the room. Hence, looking only at the persons and the room, there is no inherent structure that could be used for predefined rule based disclosure models. This shall emphasize the in situ characteristic of information disclosure in such a meeting. 


\section{Overview}

The next section presents related work on user side privacy control in smart environments. As a result of this review we motivate an ad hoc approach of privacy management in ubiquitous computing environments. This ad hoc approach is described in section 3 . An ad hoc privacy control requires a significant portion of a user's attention to focus on privacy issues additionally to the actual task to fulfil within the environment. To reduce the necessary user attention we are developing an assistance for deciding the disclosure of personal information based on learning techniques. The general idea of such a learning approach is described in detail in one of our previous work 1. A crucial aspect of a disclosure assistance is how users can interact with the assistance. The data managed by the assistance may be very sensitive. Thus in most cases users want to be able to understand and control automated disclosure decisions. In section 4 we present different approaches for user interaction with a disclosure assistance. The critical point here is the transparency of the assistance, i.e. how users can verify and align automated ad hoc disclosure decision with actual privacy preferences. Finally, in section 5, we summarize our work and provide an outlook on future work.

\section{Related Work}

Typical approaches to control personal information disclosure are rule and role based systems. Users can set up rules to decide conditions for disclosing an information and roles for specifying the information to disclose. Alternatively the terms policy (for a rule set) and identity or face (for a role) are used.

An example for a policy based approach is given by Langheinrich [5]. He presents pawS, a privacy awareness system that extends P3P/APPEL11 for ubiquitous systems. Services within a smart environment announce their data handling policy expressed in $\mathrm{P} 3 \mathrm{P}$ via service related privacy proxies. In return, users express privacy preferences using APPEL and personal privacy proxies. These proxies then negotiate the flow of personal information between a user and services within the environment. It is advantageous that this approach makes use of already existent concepts. On the other side, while there are user friendly tools for specifying privacy preferences and interacting with service-side policies in the realm of web activities [3], there is a lack of such tools for the domain of ubiquitous systems. Further pawS deals with privacy issues concerning the used infrastructure - our work focuses on interpersonal privacy implications.

In the domain of location privacy, Myles et al. 9] suggest a rule based system to decide disclosure of location information (also partly using P3P/APPEL). Location information is shared by a location server which contacts user specific validators if someone requests a user's location. Amongst other methods, these validators decide the location disclosure based on a set of rules defined by its users. The authors suggest that location provider offer appropriate rule set templates and additionally "wizards" help users to comfortably set up rules that

\footnotetext{
${ }^{1}$ Specifications of P3P and APPEL can be found at http://www.w3.org/TR/
} 
differ from the templates. This approach limits its use for managing location. It could be extended for other types of personal information but that would add complexity to the rule sets users have to specify in their validators. Actually Prabaker et al. 11] have shown that users already have difficulties to set up disclosure rules for location as the only type of information to manage.

Next to rules, there exist concepts which utilize virtual roles, identities 2418 or faces [7] for managing disclosure of personal information in smart environments. The basic idea is to abstract a specific set of personal information to a role, e.g. "anonymous", "private", "job" or "public". Roles are supposed to provide an easy to grasp way of managing personal information. However, role concepts always conflict between simple but too general and subtle but too complex. In fact Lederer highlights this problem of generality in a subsequent work 6 .

Rule and role based approaches to control communication of personal information try to release users from repeatedly deciding information disclosure ad hoc. On the other side such preconfigured privacy fails in many scenarios as it contradicts to the way users normally practise privacy. Rules require users to specify their privacy preferences in advance in an abstract manner. As long as users have a clear idea of situations to come and how to handle their information in these situations rules and roles are a suitable way for managing personal information. In contrast when situations get more complex and the diversity of potentially communicated personal information grows or is not known in advance, policies are hard to create and maintain 116. Roles, as a concept for managing the possible sets of information to disclose, fail if a user's privacy preferences do not match a clear scheme like "private", "job" or "public" but requires a more fine-grained selection of information. In that case the increased number of roles would make it hard for users to distinguish and maintain their roles.

The main message of this review is that existent work on user side control of personal information disclosure in smart environments focuses on a priori configured privacy preferences, which conflicts with the dynamic and intuitive aspects of privacy. There are cases when the decision which information to communicate within a smart environment can only be decided ad hoc, in the moment of disclosure: when the number of possible situations is very high, when there are situations which cannot be predicted or when there are many, subtly differing variances in the personal information to disclose.

\section{Ad Hoc Privacy Management}

An ad hoc approach of controlling the flow of personal information in smart environments has some significant advantages. It releases users from drawing attention to privacy before the actual use of a system. Instead users decide the disclosure of their information in the moment when it is utilized by an environment. Hence, users are able to perceive the immediate implications of information disclosure and can perform privacy in conformance with intuitive and up to date preferences. Obviously a disadvantage is that a significant portion of a user's attention, while interacting with services, is needed for privacy issues, which reduces the attention a user can draw on the actual task to fulfil with the service. 


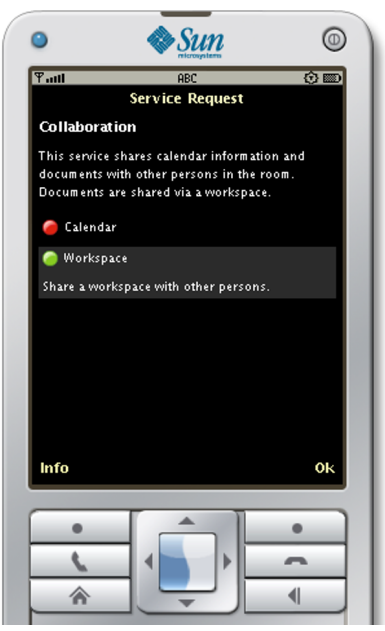

(a)

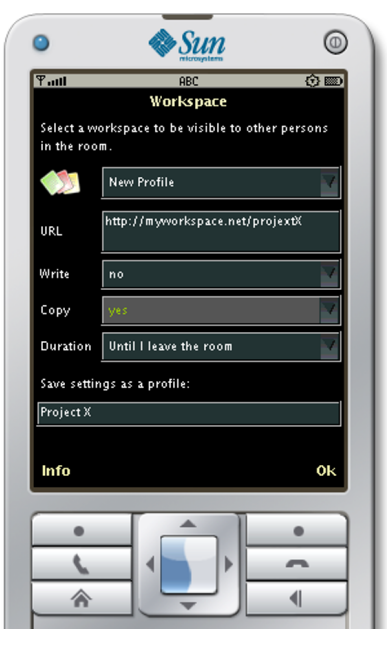

(b)

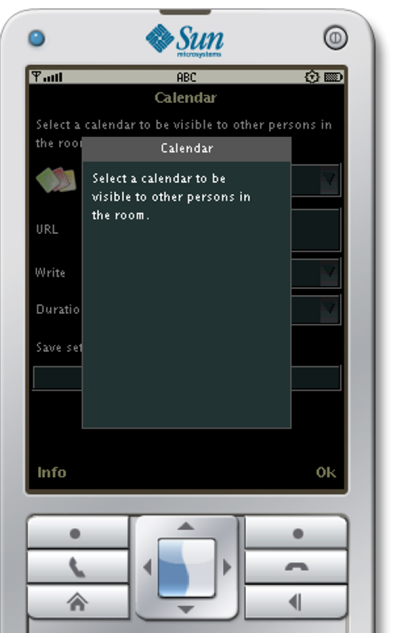

(c)

Fig. 2. Users decide the disclosure of personal information with their mobile devices during service interaction. A service lists its required personal information (a). For each information users are able to configure a disclosure for the requested information (b). Detailed meta information for the service in general and each requested information informs users about privacy implications when using this service (c).

In other words, an ad hod privacy control is more similar to natural privacy handling but it may overload a user's attention. To balance this drawback an assistance is needed, that relieves users of frequently making disclosure decisions while still supporting the general idea of ad hoc privacy management. For this purpose we suggest a learning scheme that observes and learns a user's decisions which information to disclose to which service in which situation (respectively context). Currently we are doing a user study which observes context and disclosure behavior of users in a virtual smart environment. Virtual means that users can discover and interact with services to negotiate communication of personal information, but the services' proposed functionality is not present as it is not part of our observations. This enables us to be as flexible as possible in setting up service environments for our observation scenarios. Figure 2 illustrates how users interact with services in the context of ad hoc privacy management.

First observation results have shown, that it is possible to correlate user disclosure decisions with context information 2. Examples for context information utilized in our setting are nearby persons, devices and services, time (day of week, hour of day, ...), location and movement patterns of persons within the environment. The intended result of a learning scheme is to generalize this correlation to a disclosure decision model (DDM) that "mirrors" a user's privacy preferences and that can be used as an agent managing private data on behalf of

${ }^{2}$ Complete results and their analysis are part of a separate work, submitted for publication at the Symposium On Usable Privacy and Security, SOUPS 2009. 
the user (or at least making suggestions for disclosure decisions) [1]. The challenges on learning a users disclosure behavior are input selection (determining disclosure-relevant context information and its levels of abstraction), learner implementation (choosing an appropriate method with regard to accuracy) and output representation (a user's view on and interaction with a learned output model). The challenge focused here is the output representation, i.e. how users see and interact with a DDM.

A DDM maps a service, its requested information and the context during the request to a disclosure decision. We use the following notation for this mapping. A service $S$ requests several information labeled $I_{1}, . ., I_{n}$. For each information $I_{i}$ there is a set of possible disclosure configurations $D\left(I_{i}\right)=I_{i}^{1}, . ., I_{i}^{m}$. So $D\left(I_{i}\right)$ describes all configurations specified by a user for an information $I_{i}$. The subset $D_{S}\left(I_{i}\right) \subseteq D\left(I_{i}\right)$ describes all disclosure configurations of $I_{i}$ previously used for the service $S$. The context $C$ during the request is composed by several context information $c_{i}$ so that $C=\left(c_{i}, . ., c_{m}\right)$. Given that, we express a user's disclosure behavior as follows:

$$
S \times I_{1} \times . . \times I_{n} \times C \rightarrow D_{S}\left(I_{1}\right) \times \ldots D_{S}\left(I_{n}\right)
$$

This maps a service $S$, its requested information $I_{i}$ and a context $C$ to disclosure configurations $D_{S}\left(I_{i}\right)$. For now disclosure configurations are simply considered as instances, we do not regard their inner structure. Future work may also deal with integrating the inner parameters of a disclosure configuration (as seen in Fig. 2,b) into the modeling of disclosure decisions.

\section{User Interaction with DDMs}

In an ideal case the mapping (1) describes privacy preferences in such a way that users are able to match and align them with real preferences (Fig. 3. a). However, this requires a rather small set of disclosure rules. Further, these rules must be expressed with an appropriate abstraction of the context information. Low level context like raw sensor values do not match intuitive privacy preferences which utilize user selected abstract situation characteristics. These requirements on the disclosure model representation limit the learning schemes that can be used to create DDMs and may drop learning schemes which are less transparent but provide better performance. Further the required context abstraction (done by the developer) is a first error source for DDMs, as there is no general abstraction valid for all users. This motivates to also consider learning schemes that create non-transparent DDMs. Since no learning scheme will create a perfect DDM, the question arises, how users can interact with black box DDMs when they cannot control a DDM's inner decision process.

The simplest approach to adjust a black box DDM are user vetoes which mark wrong disclosure decisions (Fig. 3.b). This is simply continued learning. In this case users are only able to react, they cannot integrate distinct preferences into a DDM which have not yet been learned by the DDM's learning scheme. Such a DDM can only be used as a suggestion mechanism. To automate disclosure 


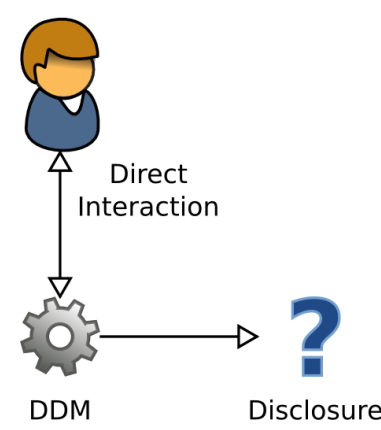

a) Transparent DDM

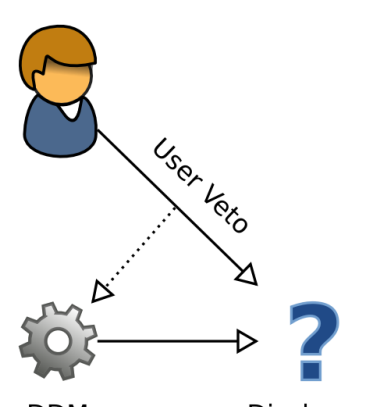

DDM

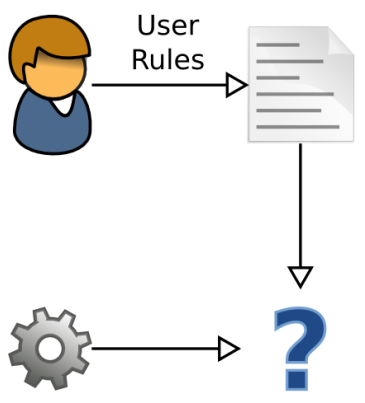

DDM

Disclosure

c) Black box + user rules

Fig. 3. User interaction with DDMs depends on the transparency of a DDM's internal decision process

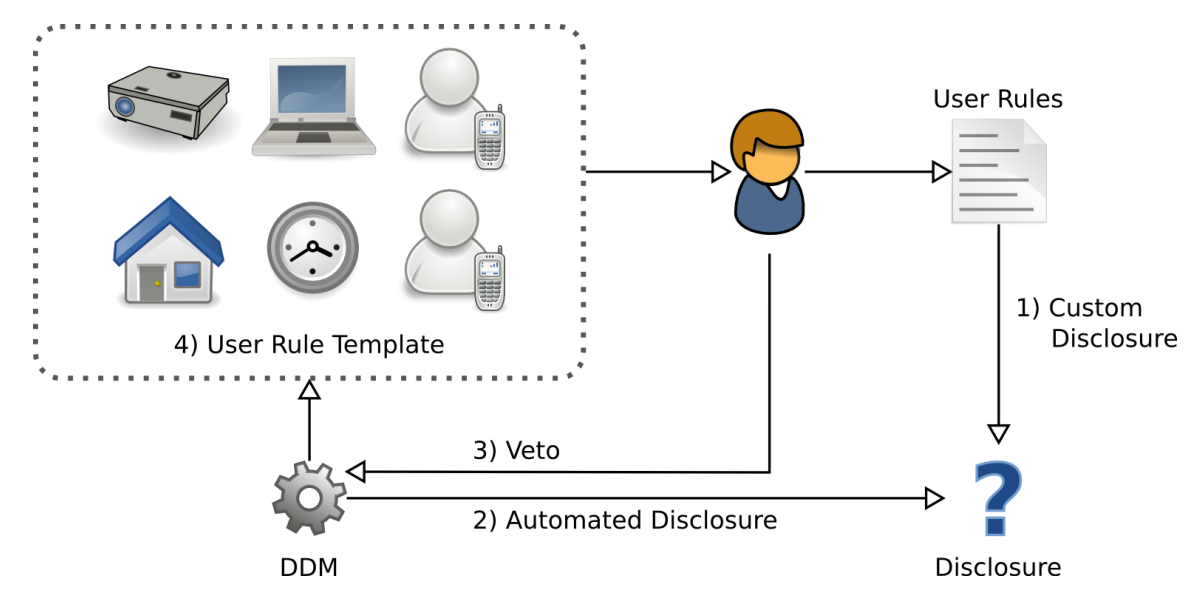

Fig. 4. A disclosure process integrating a black box DDM, user vetoes and manual user rules. User rules have a higher priority than DDMs to enable users to express clear disclosure decisions for sensitive personal information. A DDM assists the creation of user rules by providing relevant context information for rule parametrization.

decisions, users must be sure that certain sensible information is handled properly by the DDM. Since a black box DDM cannot be adjusted in that manner, it could be backed up by a simple set of disclosure rules manually compiled by users (Fig. 3. c). In fact, a veto based interaction can be combined with a black box DDM backed up by user rules. Whenever users express a veto on a disclosure decision suggested by a DDM, users can be supported in specifying a manual disclosure rule, e.g. by providing the current context information that can be used to parametrize a manual disclosure rule.

Figure 4 illustrates the combination of user vetoes and user rules. Here, the first step in deciding a disclosure is to query the user rules, they have priority 
over disclosure decisions of the DDM. If the user rules do not apply to the current situation, the DDM is consulted (step 2). At this point users can express a veto if they do not feel comfortable with the automated disclosure decision (step 3). As a result, the DDM hands out a template for a custom rule to set up by the user (step 4). This template is a draft for a rule using current context information and the requested information as parameters. Setting up custom disclosure rules in that manner preserves the aimed ad hoc characteristic of privacy management, it releases users from abstracting privacy preferences to rules a priori.

\section{Conclusion and Outlook}

In this work we discussed the management of private information concerning interpersonal privacy implications in smart environment. We identified limitations in existing work, which mostly does not match the intuitive and dynamic aspects of privacy. As an alternative we presented an ad hoc approach to privacy management. This approach uses a disclosure assistance based on learning techniques. To enable users to validate and align actual privacy preferences with the assistance, we presented two concepts for the interaction between a user and a disclosure assistance. Currently we are accumulating user disclosure data which is used for implementing a learning scheme as described above. In our future work we use this learning scheme for implementing and evaluating the presented approaches for interacting with the disclosure assistance.

\section{References}

1. Bünnig, C.: Learning context based disclosure of private information. In: The Internet of Things \& Services - 1st Intl. Research Workshop, Valbonne, France (September 2008)

2. Clauß, S., Pfitzmann, A., Hansen, M.: Privacy-enhancing identity management. The IPTS Report 67, 8-16 (September 2002)

3. Cranor, L.F., Guduru, P., Arjula, M.: User interfaces for privacy agents. ACM Trans. Comput.-Hum. Interact. 13(2), 135-178 (2006)

4. Jendricke, U., Kreutzer, M., Zugenmaier, A.: Pervasive privacy with identity management. Technical Report 178, Universität Freiburg (October 2002)

5. Langheinrich, M.: A privacy awareness system for ubiquitous computing environments. In: Borriello, G., Holmquist, L.E. (eds.) UbiComp 2002. LNCS, vol. 2498, p. 237. Springer, Heidelberg (2002)

6. Lederer, S., Hong, J.I., Dey, A.K., Landay, J.A.: Personal privacy through understanding and action: Five pitfalls for designers. Personal Ubiquitous Computing 8(6), 440-454 (2004)

7. Lederer, S., Mankoff, J., Dey, A.K., Beckmann, C.P.: Managing personal information disclosure in ubiquitous computing environments. Technical Report UCB/CSD-03-1257, University of California, Berkeley (2003)

8. Maibaum, N., Sedov, I., Cap, C.H.: A citizen digital assistant for e-government. In: Traunmüller, R., Lenk, K. (eds.) EGOV 2002. LNCS, vol. 2456, pp. 284-287. Springer, Heidelberg (2002) 
9. Myles, G., Friday, A., Davies, N.: Preserving privacy in environments with locationbased applications. IEEE Pervasive Computing 2(1), 56-64 (2003)

10. Palen, L., Dourish, P.: Unpacking "privacy" for a networked world. In: CHI 2003: Proc. of the SIGCHI Conference on Human Factors in Computing Systems, pp. 129-136. ACM, New York (2003)

11. Prabaker, M., Rao, J., Fette, I., Kelley, P., Cranor, L., Hong, J., Sadeh, N.: Understanding and capturing people's privacy policies in a people finder application. In: UBICOMP 2007: Workshop on UBICOMP Privacy (September 2007) 